\title{
Hydrodynamic Coulomb drag of strongly correlated electron liquids
}

\author{
S. S. Apostolov, ${ }^{1}$ A. Levchenko, ${ }^{1}$ and A. V. Andreev ${ }^{2}$ \\ ${ }^{1}$ Department of Physics and Astronomy, Michigan State University, East Lansing, Michigan 48824, USA \\ ${ }^{2}$ Department of Physics, University of Washington, Seattle, Washington 98195, USA
}

(Dated: September 16, 2021)

\begin{abstract}
We develop a theory of Coulomb drag in ultraclean double layers with strongly correlated carriers. In the regime where the equilibration length of the electron liquid is shorter than the interlayer spacing the main contribution to the Coulomb drag arises from hydrodynamic density fluctuations. The latter consist of plasmons driven by fluctuating longitudinal stresses, and diffusive modes caused by temperature fluctuations and thermal expansion of the electron liquid. We express the drag resistivity in terms of the kinetic coefficients of the electron fluid. Our results are nonperturbative in interaction strength and do not assume Fermi-liquid behavior of the electron liquid.
\end{abstract}

PACS numbers: 71.27.+a, 72.10.-d, 73.40.Ei, 73.63.Hs

Introduction and motivation. Interaction-induced mutual friction phenomena in the electrically disconnected double quantum wells provide a uniquely sensitive probe of electronic scattering and correlations. The effect, commonly referred to as Coulomb drag (CD) $\stackrel{1,2}{, 2}$ consists of passing a drive current $I$ through one (active) layer and measuring nonlocally induced drag voltage $V_{D}$ in the other (passive) layer. In experiments, the drag resistivity $\rho_{D}=V_{D} / I$ is usually studied as a function of temperature, magnetic field, electron density, and interlayer separation ${ }^{3-\underline{8}}$ Recent measurements of $r_{D}$ in the tightly nested graphene double layers ${ }^{9,10}$ triggered resurgence of interest and new proposals for the mechanisms of $\mathrm{CD}$ effect. 11,12

The magnitude and even the mechanism of CD depend on the temperature $T$, interlayer distance $d$, properties of the disorder potential, and the strength of electronelectron interactions. The latter is characterized by the interaction parameter $r_{s}=\left(\pi n a_{B}^{2}\right)^{-1 / 2}$, where $n$ is the electron density and $a_{B}=\epsilon / m e^{2}$ is the effective Bohr radius in the material ( $\epsilon$ being the dielectric constant, hereafter $\hbar=1$ ).

In the weakly interacting regime, $r_{s} \lesssim 1$, measurements of $\rho_{D}(T)^{\underline{3}-\underline{5}}$ are in qualitative agreement with the predictions of the Fermi-liquid theory $\underline{13}-17$ Specifically, the drag resistivity is relatively small, $\rho_{D} / \rho_{Q} \propto$ $\left(k_{F} d\right)^{-\alpha_{d}}\left(T / E_{F}\right)^{\alpha_{T}}$, scales quadratically with the temperature at $T \ll E_{F}\left(\alpha_{T}=2\right.$ up to logarithmic corrections in the disordered case $\frac{15,16}{}$ ), and is inversely proportional to a certain power of interlayer distance $d$ $\left(\alpha_{d}=2-4\right.$ depending on the ratio between $d$ and electronic mean free path), where $k_{F}$ and $E_{F}$ are Fermi momentum and energy, respectively, and $\rho_{Q}=2 \pi / e^{2}$ is the resistance quantum. At higher temperatures, $T \sim E_{F}$, drag is enhanced by the plasmons such that $\rho_{D} / T^{2}$ has a relatively broad peak structure near the characteristic energy of the plasmon modes $\frac{18}{18}$ There are few exceptions where the CD effect was studied beyond the leading order in interlayer interaction. It was shown in Ref. 19 that interference corrections to $\rho_{D}$ originating from the third-order processes in the interlayer interaction, are increasingly important at low $T$. This is in loose qualita- tive agreement with the fact that experimental values of the drag are larger than conventional values that vanish as $T \rightarrow 0$, however, the theory of Ref. 19 still assumes relatively weak interactions.

For $r_{s} \gg 1$ Coulomb drag is not well understood. In this case apart from the Fermi energy $E_{F}$ there are two other important energy scales: the interaction energy $V=r_{s} E_{F}$ and the Debye frequency $\Omega_{D} \sim \sqrt{V E_{F}}=$ $\sqrt{r_{s}} E_{F}$. As a consequence of this hierarchy $E_{F} \ll \Omega_{D} \ll$ $V$ there is a wide temperature interval, $E_{F}<T<V$, in which the system remains strongly correlated but the Fermi-liquid description does not apply. It is worth noting that such strongly correlated liquids may be treated classically only for $T>\Omega_{D}$, while at lower temperatures they form a semiquantum state $\underline{20}^{22}$ Microscopic theory of electron transport in this very interesting regime has not been developed.

In samples with $r_{s} \gg 1$ even at low temperatures, $T \ll E_{F}$, the drag resistance is one to two orders of magnitude larger than expected on the basis of a simple extrapolation of the small $r_{s}$ results $\underline{\underline{8}}$ Furthermore, the power exponent $2<\alpha_{T}<3$ deviates from its nominal value and the system has anomalous response in a magnetic field $\stackrel{8}{-}$ A detailed study based on an extrapolation of Fermi-liquid-based formulas to the region where $r_{s} \gtrsim 1$ has been carried out in Ref. 23 in an attempt to address the data of Ref. 8. Finally two elegant phenomenological theories designed for the strongly disordered electronic systems ${ }^{24}$ and electronic microemulsions ${ }^{25}$ have been proposed.

Most of the previous theoretical work on CD focused on the collisionless regime, in which the spacing between the layers is smaller than the mean free path of the quasiparticles. In this paper we develop a theory of Coulomb drag in the opposite regime, where the density fluctuations of the electron liquid responsible for Coulomb drag may be described using the hydrodynamic approach. A hydrodynamic theory of resistivity was recently formulated in Ref. 26. We generalize this theory to the case of drag resistivity in double layer systems. This requires consideration of the fluctuation corrections to hydrodynamics $\stackrel{27,28}{2}$ We identify a mechanism of drag resistivity 
originating from the entropy fluctuations, which due to the thermal expansion changes the electron density thus leading to the Coulomb coupling between the layers. The contribution from the plasmon modes is also discussed in details.

We assume that the interlayer distance exceeds the equilibration length of the electron liquid, $d \gg \ell_{\mathrm{ee}}$, and at the same time the phonon contribution to drag is negligible. Hydrodynamic theory of phonon-mediated drag for charge neutral liquids was developed in Ref. 29. The hydrodynamic description applies to any liquid type. Microscopic properties of the liquid manifest themselves via the temperature and density dependence of the kinetic coefficients. Experimentally, the hydrodynamic regime is likely to be relevant to clean low carrier density systems, e.g., hole systems of Refs. [8,30] with the typical $r_{s} \sim 10-40$ and Fermi energies on the order of Kelvin. In this case, at $T \gtrsim E_{F}$, electron-phonon scattering is still weak $\stackrel{30}{ }$ On the other hand, carriers form a nondegenerate strongly correlated liquid (semiquantum or classical), and the hydrodynamic description applies from very short distances of the order of inter-electron spacing. Drag resistivity measurements in a high mobility quantum well with $r_{s} \sim 1$ and at large interlayer spacing $(d \sim 5000 \AA)$ have been reported in the literature ${ }^{31}$ and attributed to phonon drag. $\underline{\underline{15}}$

Stochastic Navier-Stokes equations. If the interlayer spacing $d$ exceeds the equilibration length $d \gg \ell_{\mathrm{ee}}$ Coulomb drag is dominated by hydrodynamic density fluctuations. The latter obey Gaussian distribution and may be described by introducing stochastic Langevin forces into the hydrodynamic equations $\frac{27}{2}$ Having in mind linear response theory we start from the linearized continuity, Navier-Stokes and entropy production equations for a fluid moving in an external potential $U$,

$$
\begin{aligned}
& \partial_{t} \delta n+\partial_{i}\left(\delta n v_{i}+n \delta v_{i}\right)=0, \\
& m n\left(\partial_{t}+\mathbf{v} \cdot \nabla\right) \delta v_{i}=-n \partial_{i} U-\partial_{i} \delta P+\partial_{k} \delta \sigma_{i k}, \\
& n T\left(\partial_{t}+\mathbf{v} \cdot \nabla\right) \delta s=-\operatorname{div} \mathbf{Q},
\end{aligned}
$$

where $i, k$ are Cartesian indices and we used shorthand notation for the spatial derivative $\partial_{i}=\partial / \partial x_{i}$. In Eqs. (11)-(3) $n, m$, and $T$ are, respectively, the equilibrium particle density, mass, and temperature; $\mathbf{v}$ is the uniform fluid velocity; while $\delta n, \delta v_{i}, \delta s$, and $\delta P$ are fluctuations of density, velocity, entropy per particle, and pressure in the liquid flow. In a charged liquid, the external potential $U$ is determined by the fluid density via the Poisson equation. The set of these equations has to be replicated for both active and passive layers and we will use subscripts $\uparrow \downarrow$ to distinguish the two. The linearized viscous stress tensor $\delta \sigma_{i k}$ and the thermal energy flux $\mathbf{Q}$ are given, respectively, by

$$
\begin{aligned}
& \delta \sigma_{i k}=\eta\left(\partial_{k} \delta v_{i}+\partial_{i} \delta v_{k}\right)+(\zeta-\eta) \delta_{i k} \operatorname{div} \delta \mathbf{v}+\varsigma_{i k}, \\
& \mathbf{Q}=-\kappa \nabla \delta T+\mathbf{g} .
\end{aligned}
$$

Here $\kappa$ is the thermal conductivity, $\eta$ is the first (shear) viscosity of the liquid, and $\zeta$ is the second (bulk) viscosity. We consider a symmetric setup in which fluctuating
Langevin heat and stress fluxes in the two layers have identical variances:

$$
\begin{aligned}
& \left\langle\mathbf{g}_{i}(\mathbf{r}, t) \mathbf{g}_{j}\left(\mathbf{r}^{\prime}, t^{\prime}\right)\right\rangle=2 \kappa T^{2} \delta_{i j} \delta\left(\mathbf{r}-\mathbf{r}^{\prime}\right) \delta\left(t-t^{\prime}\right), \\
& \left\langle\varsigma_{i k}(\mathbf{r}, t) \varsigma_{l m}\left(\mathbf{r}^{\prime}, t^{\prime}\right)\right\rangle=2 T \delta\left(\mathbf{r}-\mathbf{r}^{\prime}\right) \delta\left(t-t^{\prime}\right) \\
& \times\left[\eta\left(\delta_{i l} \delta_{k m}+\delta_{i m} \delta_{k l}\right)+(\zeta-\eta) \delta_{i k} \delta_{l m}\right]
\end{aligned}
$$

where $\langle\ldots\rangle$ denotes averaging over the thermal fluctuations. The steady current $\propto n \mathbf{v}$ in the active layer exerts the drag force $\mathbf{F}_{D}=\left\langle\delta n_{\downarrow}\left(-\nabla U_{\downarrow}\right)\right\rangle$ on the passive layer. Relating the potential to density fluctuations by using the Poisson equation and ignoring the intralayer forces we can express the drag force in terms of the densitydensity correlation function

$$
\mathbf{F}_{D}=\sum_{q, \omega}(-i \mathbf{q}) \frac{2 \pi e^{2}}{\epsilon q} e^{-q d}\left\langle\delta n_{\uparrow}(\mathbf{q}, \omega) \delta n_{\downarrow}(-\mathbf{q},-\omega)\right\rangle
$$

where $\delta n_{\uparrow, \downarrow}(\mathbf{q}, \omega)$ are the Fourier components of the density fluctuations in both layers and $q$ is the absolute value of the vector $\mathbf{q}$. Knowing the drag force one readily finds the drag resistivity $\rho_{D}=\mathbf{F}_{D} / \mathbf{v} e^{2} n^{2}$.

Results for the drag resistivity. Our technical goal now is to solve coupled equations (11)-(3) to the linear order in $\mathbf{v}$. It will be convenient for our purposes to choose entropy and density as independent variables, and thus express temperature and pressure fluctuations via thermodynamic relations. To this end, we rewrite Eqs. (1)(33) in the Fourier components, relate temperature fluctuations to the entropy and density $\delta T=(\partial T / \partial s)_{V} \delta s+$ $(\partial T / \partial n)_{S} \delta n$, and exclude $\delta \mathbf{v}$ with the help of the continuity equation (11). We thus find for the active layer

$$
\left(\nu q^{2}-i \omega+i \mathbf{q v}\right)(\omega-\mathbf{q} \mathbf{v}) \delta n_{\uparrow}=-\frac{i q^{2}}{m}\left(\delta P_{\uparrow}+n U_{\uparrow}\right)+\frac{i \mathbf{q}}{m}\left(\hat{\varsigma_{\uparrow}} \mathbf{q}\right),
$$

$$
\left(\chi q^{2}-i \omega+i \mathbf{q v}\right) \delta s_{\uparrow}+\chi q^{2}\left(\frac{\partial s}{\partial n}\right)_{T} \delta n_{\uparrow}=-\frac{i \mathbf{q g}_{\uparrow}}{n T} .
$$

Here $\nu=(\eta+\zeta) / m n$ is the sum of shear and bulk kinematic viscosities and $\chi=\kappa / n c_{v}$ is the thermal diffusivity, while $c_{v}=T(\partial s / \partial T)_{V}$ is the heat capacity. In the passive layer we have the same set of equations but with $\mathbf{v}=0$ and an interchange of indices $\uparrow \rightarrow \downarrow$. It becomes apparent from the structure of Eqs. (9a) and (9b) that even though we consider a disorder-free system the entropy fluctuations in the liquid propagate diffusively. Because of thermal expansion, they result in diffusively spreading density fluctuations that occur at uniform stress in the liquid. The pressure fluctuations $\delta P$ can be expressed in terms of density and entropy in a given layer, while the external potential $U$ is determined by the density fluctuations in both layers. Specifically we have for the active layer

$$
\begin{array}{r}
\delta P_{\uparrow}+n U_{\uparrow}=\left(\frac{\partial P}{\partial s}\right)_{V} \delta s_{\uparrow}+\left(\frac{\partial P}{\partial n}\right)_{S} \delta n_{\uparrow} \\
+\frac{2 \pi n e^{2}}{\epsilon q}\left(\delta n_{\uparrow}+e^{-q d} \delta n_{\downarrow}\right),
\end{array}
$$


whereas the pressure variation in the passive layer $\delta P_{\downarrow}$ is obtained from above by interchanging indices $\uparrow \rightleftarrows \downarrow$. At wavelengths longer than the screening length the second term in the right-hand side of Eq. (10) is small in comparison to the third and can be neglected. Physically this means that the dependence of the stress on the density of the electron liquid is dominated by the long-range Coulomb interaction. At the same time, the first term in the right-hand side of Eq. (10) must be retained because it describes the dependence of the stress on the different thermodynamic variable, $\delta s$. With the aid of Eq. (10) we can exclude entropy fluctuations from Eqs. (9a) and (9b), and thus arrive at two coupled linear algebraic equations for the variances of thermally induced density fluctuations between the layers

$$
\begin{gathered}
\Pi_{ \pm} \delta n_{ \pm}=\frac{i \mathbf{q v}}{2}\left[\Gamma_{+} \delta n_{+}+\Gamma_{-} \delta n_{-}-\frac{\mathbf{q}}{m}\left(\hat{\varsigma}_{+} \mathbf{q}\right)-\frac{\mathbf{q}}{m}\left(\hat{\varsigma}_{-} \mathbf{q}\right)\right] \\
-\frac{i q^{2}}{m c_{v}}\left(\frac{\partial s}{\partial \ln n}\right)_{T}\left(\mathbf{q g}_{ \pm}\right)-\left(\omega_{\chi}-i \omega\right) \frac{\mathbf{q}}{m}\left(\hat{\varsigma}_{ \pm} \mathbf{q}\right), \quad(11)
\end{gathered}
$$

where we introduced symmetric $(+)$ and antisymmetric (-) combinations of the fields $\delta n_{ \pm}=\delta n_{\uparrow} \pm \delta n_{\downarrow}$, and similarly for all other quantities. The propagator of the excitation modes $\Pi_{ \pm}$and the vertex function $\Gamma_{ \pm}$are defined by the following expressions

$$
\Pi_{ \pm}(\mathbf{q}, \omega)=\left(\omega_{\nu}-i \omega\right)\left(\omega_{\chi}-i \omega\right) i \omega-\left(\omega_{\chi}-i \omega\right) \omega_{ \pm}^{2}-\omega_{\chi} \omega_{\alpha}^{2},
$$

$$
\Gamma_{ \pm}(\mathbf{q}, \omega)=\left(\omega_{\nu}-2 i \omega\right)\left(\omega_{\chi}-2 i \omega\right)+\left(\omega^{2}+\omega_{ \pm}^{2}\right) .
$$

Here we introduced characteristic mode frequencies: $\omega_{\chi}=\chi q^{2}$ and $\omega_{\nu}=\nu q^{2}$ correspond to the thermal and viscous diffusion, the frequencies $\omega_{ \pm}^{2}=\omega_{p}^{2}\left(1 \pm e^{-q d}\right)$, with $\omega_{p}^{2}=2 \pi e^{2} n q / \epsilon m$, correspond to the plasmons, while $\omega_{\alpha}=u q$ corresponds to the acoustic mode associated with the thermal expansion of the fluid with the characteristic velocity $u=\sqrt{T / m c_{v}}(\partial s / \partial \ln n)_{T}$. In deriving Eq. (11) we also made use of the thermodynamic relation $(\partial P / \partial s)_{V}=n^{2}(\partial T / \partial n)_{S}$. We look for the solution of Eq. (11) to the linear order in $\mathbf{v}$ in the form $\delta n_{ \pm}=\delta n_{ \pm}^{(0)}+\delta n_{ \pm}^{(1)}$ where

$$
\delta n_{ \pm}^{(0)}=-\frac{1}{\Pi_{ \pm}}\left[\frac{i u q^{2}}{\sqrt{m c_{v} T}}\left(\mathbf{q g}_{ \pm}\right)+\left(\omega_{\chi}-i \omega\right) \frac{\mathbf{q}}{m}\left(\hat{\varsigma}_{ \pm} \mathbf{q}\right)\right]
$$

$\delta n_{ \pm}^{(1)}=\frac{i(\mathbf{q v})}{2 \Pi_{ \pm}}\left[\Gamma_{+} \delta n_{+}^{(0)}+\Gamma_{-} \delta n_{-}^{(0)}-\frac{\mathbf{q}}{m}\left(\hat{\varsigma}_{+} \mathbf{q}\right)-\frac{\mathbf{q}}{m}\left(\hat{\varsigma}_{-} \mathbf{q}\right)\right]$

Having found $\delta n$ we are in a position to compute the density-density correlation function that determines the drag force in Eq. (8). For this purpose we use thermal averages $\left\langle\mathbf{q}\left(\hat{\varsigma}_{ \pm} \mathbf{q}\right) \mathbf{q}\left(\hat{\varsigma}_{ \pm} \mathbf{q}\right)\right\rangle=4 T q^{4}(\eta+\zeta)$ and $\left\langle\left(\mathbf{q g}_{ \pm}\right)\left(\mathbf{q g}_{ \pm}\right)\right\rangle=4 \kappa q^{2} T^{2}$, which follow directly from the
Langevin heat flux variances, and find

$$
\begin{gathered}
\left\langle\delta n_{\uparrow}(\mathbf{q}, \omega) \delta n_{\downarrow}(-\mathbf{q},-\omega)\right\rangle=2 \operatorname{in}(\mathbf{q} \mathbf{v}) \frac{q^{2}}{m} T\left(\omega_{+}^{2}-\omega_{-}^{2}\right) \\
\times \frac{\omega_{\nu}^{2} \omega^{4}+2 \omega_{\nu} \omega_{\chi}\left(\omega_{\nu} \omega_{\chi}+2 \omega_{\alpha}^{2}\right) \omega^{2}+\omega_{\chi}^{2}\left(\omega_{\nu}^{2} \omega_{\chi}^{2}-\omega_{\alpha}^{4}\right)}{\left|\Pi_{+}\right|^{2}\left|\Pi_{-}\right|^{2}} .(14)
\end{gathered}
$$

Before performing frequency integration in Eq. (8) with the density correlator from Eq. (14) we need to analyze the structure of poles of the propagator $\Pi_{ \pm}$. Under the physically relevant simplifying condition $\omega_{\nu, \chi} \ll$ $\min \left\{\omega_{\alpha}, \omega_{ \pm}\right\}$, which is justified by the fact that typical momentum transferred between the layers is small $q \sim d^{-1} \ll \sqrt{n}$, we see that $\left.\Pi_{ \pm}\right|_{\omega_{\chi}=0}=-i(\omega+i 0)\left(\omega^{2}+\right.$ $\left.i \omega \omega_{\nu}-\omega_{ \pm}^{2}\right)$. Already at this level we can identify plasmon poles at energies $\omega_{ \pm}$whose imaginary part (lifetime) is governed by the diffusive viscous mode $\omega_{\nu}$. Since $\omega_{\nu} \propto q^{2}$ fluctuations with sufficiently low momenta have arbitrary large mean free path, and therefore plasmons are well defined excitations. At the finite but small $\omega_{\chi}$ we can identify another pole, $\omega+i 0 \rightarrow \omega+i \omega_{\chi}\left(1+\omega_{\alpha}^{2} / \omega_{ \pm}^{2}\right)$, which is governed by the thermal diffusion mode. We conclude that density fluctuations that belong to one of the two parametrically distinct frequency ranges $\omega \sim \omega_{\chi}$ and $\omega \sim \omega_{ \pm}$give the largest contribution to the drag force. Integrating Eq. (8) within the leading pole approximation we find our main result for the drag resistivity

$$
\rho_{D}=\frac{1}{16 \pi^{2} e^{2}} \frac{1}{n d^{2}}\left[\frac{T}{\chi n} \mathcal{F}_{1}(\alpha)+\frac{2 \pi \nu T}{\varpi^{2} n d^{4}} \mathcal{F}_{2}(\beta)\right],
$$

where $\varpi=\sqrt{2 \pi e^{2} n / \epsilon m d}$ is the plasmon energy taken at the wave vector corresponding to the interlayer separation. Two dimensionless parameters here are

$$
\alpha=\left(\frac{u}{\varpi d}\right)^{2}, \quad \beta=\left(\frac{\nu}{\varpi d^{2}}\right)^{2},
$$

and the two respective dimensionless functions are defined by the following momentum integrals in the rescaled units

$$
\begin{aligned}
& \mathcal{F}_{1}(\alpha)=\int_{0}^{\infty} \frac{4 \pi \alpha^{2} x^{3} e^{-2 x} \mathrm{~d} x}{\left[(\alpha x+1)^{2}-e^{-2 x}\right]\left[(\alpha x+1)-e^{-2 x}\right]} \\
& \mathcal{F}_{2}(\beta)=\int_{0}^{\infty} \frac{2 x^{4}\left(\beta x^{3}+1\right) e^{-2 x} \mathrm{~d} x}{\left[1-e^{-2 x}\right]\left[\beta x^{3}+e^{-2 x}\right]}
\end{aligned}
$$

When deriving above expressions we used $\left(\omega_{+}^{2}-\right.$ $\left.\omega_{-}^{2}\right) / \omega_{+}^{2} \omega_{-}^{2}=\left(\omega_{p}^{2} \sinh (q d)\right)^{-1}$, and rescaled momentum integration in the units of the interlayer distance $(x=$ $q d)$. The first term in Eq. (15) stems from the slow thermal modes and is inversely proportional to the thermal conductivity, while the second one is due to plasmons. We stress that Eq. (15) represents a nonperturbative in interaction result for the drag resistivity and as such applies to electron bilayers with $r_{s} \gg 1$. The functions $\mathcal{F}_{1,2}$ are plotted in Fig. (11) and they are almost constants of the order of unity $\mathcal{F}_{1,2} \sim 1$ in a wide parameter range $\{\alpha, \beta\} \gtrsim 1$. For $\{\alpha, \beta\} \ll 1$, which is most likely relevant 


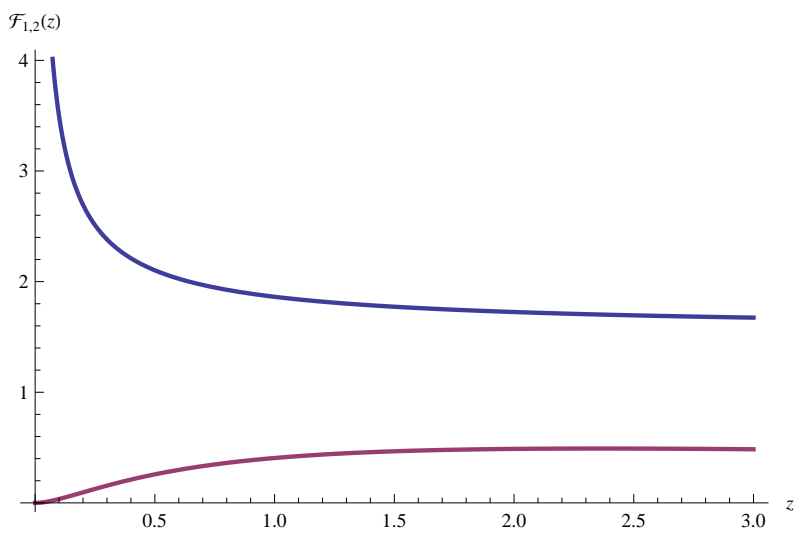

FIG. 1: Dimensionless functions $\mathcal{F}_{1}$ (bottom line) and $\mathcal{F}_{2}$ (top line) that enter the drag resistivity are plotted versus their respective scaling variable $z=\alpha$ and $z=\beta$, see Eqs. (15)(18) for the definition.

to experiments, one easily finds that $\mathcal{F}_{1} \approx 3 \pi \zeta(3) \alpha^{2} / 2$ and with the logarithmic accuracy $\mathcal{F}_{2} \approx \frac{1}{8} \ln ^{4}(1 / \beta)$, so that Eq. (15) can be simplified to

$$
\begin{aligned}
& \frac{\rho_{D}}{\rho_{Q}} \simeq \frac{3 \varsigma[3] \epsilon^{2} T^{3}}{128 \pi^{3} e^{4} \kappa c_{v} n^{3} d^{4}}\left(\frac{\partial s}{\partial \ln n}\right)_{T}^{4} \\
& +\frac{\epsilon T(\eta+\zeta)}{128 \pi^{2} e^{2} n^{4} d^{5}} \ln ^{4}\left(\frac{2 \pi e^{2} m n^{3} d^{3}}{\epsilon(\eta+\zeta)^{2}}\right),
\end{aligned}
$$

where $\varsigma[z]$ is the Riemann zeta function. Interestingly, thermal expansion and plasmon mediated contributions to the Coulomb drag resistivity Eq. (19) have distinct dependencies on the electron density $n$ and interlayer separation $d$. One should also notice that complete temperature dependence of $\rho_{D}(T)$ is implicit in the corresponding temperature dependencies of the respective kinetic and thermodynamic coefficients $\kappa(T), \eta(T), \zeta(T)$ and $c_{v}(T)$. Although a detailed microscopic theory for the temperature dependence of $\kappa, \eta$ and $\zeta$ of nondegenerate strongly correlated liquids has not been developed, some conjectures were put forward in Refs. [20,33]. In particular for the semiquantum regime at $E_{F}<T<\Omega_{D}$ one estimates $c_{v} \propto T, \kappa \propto T$, and $\eta \propto 1 / T$.

In contrast, the Fermi-liquid regime has been studied extensively, $\stackrel{34}{\underline{w}}$ and for $T \lesssim E_{F}$ one readily finds $\kappa \sim c_{v} n \ell_{\mathrm{ee}} v_{F} \sim E_{F}^{2} / T, \eta \sim m v_{F} n \ell_{\mathrm{ee}} \sim n E_{F}^{2} / T^{2}$, and $c_{v} \sim T / E_{F}$. Note that for Fermi liquids the temperature dependence of the drag resistivity in the hydrodynamic regime is drastically different from the conventional $T^{2}$ law. Indeed, assuming that interaction parameter $r_{s} \sim 1$ one estimates the first term in Eq. (19) as $\sim\left(T / E_{F}\right)^{7}\left(1 / k_{F} d\right)^{4}$ and the second one as $\sim\left(E_{F} / T\right)\left(1 / k_{F} d\right)^{5}$. At $T<E_{F} /\left(k_{F} d\right)^{1 / 8}$ the second term dominates and we obtain the following estimate for the drag coefficient of Fermi liquids in the hydrodynamic regime

$$
\frac{\rho_{D}}{\rho_{Q}} \sim \frac{E_{F}}{T} \frac{1}{\left(k_{F} d\right)^{5}}
$$

The hydrodynamic description is restricted to temperatures $T>E_{F} / \sqrt{k_{F} d}$ where $\ell_{\mathrm{ee}}<d$. It is striking to observe that at temperatures where $\ell_{\mathrm{ee}} \sim d$ the hydrodynamic result above, $\rho_{D} / \rho_{Q} \sim\left(1 / k_{F} d\right)^{9 / 2}$, is parametrically larger (in $\sqrt{k_{F} d} \gg 1$ ) than the conventional FL result for the collisionless regime, $\rho_{D} / \rho_{Q} \simeq$ $\left(T / E_{F}\right)^{2} /\left(k_{F} d\right)^{4} \sim\left(1 / k_{F} d\right)^{5}$. This implies that collisions strongly enhance Coulomb drag. The study of the crossover from a collisionless to a collision-dominated regime is an interesting problem that is beyond the scope of the present work.

Discussion. It is perhaps instructive to compare our result for the drag resistivity Eq. (19) to the hydrodynamic result for intralayer resistivity in Ref. 26 [see their Eq. (6)]. Both are given by the sum of thermal and viscous terms, which have similar dependence on the viscosity and thermal conductivity of the fluid. This is not accidental. To second order in the disorder potential the intralayer resistivity can be understood in terms of the drag force between the electron liquid and the disorder potential $\mathbf{F}_{D}=\langle\delta n(-\nabla U)\rangle$ with $U$ representing the disorder potential. In that case, the fluctuations of density in the electron liquid are created by the disorder potential itself. The subsequent scattering of density fluctuations from the disorder potential produces a net resistive force. In the case of drag, both the scattering potential $U$ and the fluctuations of the electron density are produced by thermal fluctuations, whose variance depends on the temperature. This accounts for the difference between the temperature dependence of drag and intralayer resistivity. On the other hand, the propagation of fluctuations in the fluid in either case is described by the same linearized hydrodynamic equations, and occurs in the form of stress-driven ballistic modes and entropy-driven diffusive modes. This results in the similarity between the corresponding expressions.

The above qualitative arguments are useful in contrasting our theory with the energy transfer mechanism of Coulomb drag studied in the context of graphene double layers $\stackrel{11}{\underline{w}}$ The energy transfer mechanism (E-drag) does not involve thermal fluctuations, and may be treated in the main hydrodynamic approximation. On the other hand, this mechanism relies on correlations of the disorder potential in the layers and disappears in the clean limit. The contribution considered in the present paper arises from fluctuation corrections to hydrodynamics, and remains finite in the clean limit. The common feature of two mechanisms is that diffusive redistribution of thermal energy in the electron liquid plays a crucial role in supporting strong Coulomb drag.

Acknowledgments. We would like to thank B. Spivak for many useful discussions, and J. Song and L. Levitov for discussions on the energy-driven mechanism of Coulomb drag. A.L. acknowledges support from NSF under Grant No. PHYS-1066293 and the hospitality of the Aspen Center for Physics where part of this work was performed. The work of A. V. A. was supported by DOE Grant No. DE-FG02-07ER46452. 
1 M. B. Pogrebinskii, Fiz. Tekh. Poluprovodn. 11, 637 (1977) [Sov. Phys. Semicond. 11, 372 (1977)].

2 P. M. Price, Physica B (Amsterdam) 117, 750 (1983).

3 P. M. Solomon, P. J. Price, D. J. Frank, and D. C. La Tulipe, Phys. Rev. Lett. 63, 2508 (1989).

${ }^{4}$ T. J. Gramila, J. P. Eisenstein, A. H. MacDonald, L. N. Pfeiffer, and K. W. West, Phys. Rev. Lett. 66, 1216 (1991); Physica B: Condensed Matter 197, 442 (1994).

5 U. Sivan, P. M. Solomon, and H. Shtrikman, Phys. Rev. Lett. 68, 1196 (1992).

${ }^{6}$ M. P. Lilly, J. P. Eisenstein, L. N. Pfeiffer, and K. W. West, Phys. Rev. Lett. 80, 1714 (1998).

7 M. Kellogg, J. P. Eisenstein, L. N. Pfeiffer, and K. W. West, Phys. Rev. Lett. 90, 246801 (2003).

8 R. Pillarisetty, H. Noh, D. C. Tsui, E. P. De Poortere, E. Tutuc, and M. Shayegan, Phys. Rev. Lett. 89, 016805 (2002; ibid. 90, 226801 (2003).

9 S. Kim, I. Jo, J. Nah, Z. Yao, S. K. Banerjee, and E. Tutuc, Phys. Rev. B 83, 161401(R) (2011).

10 R. V. Gorbachev, A. K. Geim, M. I. Katsnelson, K. S. Novoselov, T. Tudoroskiy, I. V. Grigorieva, A. H. MacDonlad, K. Wantanabe, T. Taniguchi, and L. A. Ponomarenko, Nat. Phys. 8, 896 (2012).

11 J. C. W. Song and L. S. Levitov, Phys. Rev. Lett. 109, 236602 (2012); ibid. 111, 126601 (2013); J. C. W. Song, D. A. Abanin, L. S. Levitov, Nano Lett. 13, 3631 (2013).

12 M. Schütt, P. M. Ostrovsky, M. Titov, I. V. Gornyi, B. N. Narozhny, A. D. Mirlin, Phys. Rev. Lett. 110, 026601 (2013); M. Titov, R. V. Gorbachev, B. N. Narozhny, T. Tudorovskiy, M. Schütt, P. M. Ostrovsky, I. V. Gornyi, A. D. Mirlin, M. I. Katsnelson, K. S. Novoselov, A. K. Geim, and L. A. Ponomarenko, Phys. Rev. Lett. 111, 166601 (2013).

13 B. Laikhtman and P. M. Solomon, Phys. Rev. B 41, 9921 (1990).

14 A.-P. Jauho and H. Smith, Phys. Rev. B 47, 4420 (1993).

15 L. Zheng and A. H. MacDonald, Phys. Rev. B 48, 8203 (1993).

16 A. Kamenev and Y. Oreg, Phys. Rev. B 52, 7516 (1995).

17 K. Flensberg, B. Y.-K. Hu, and A.-P. Jauho and J. M. Kinaret, Phys. Rev. B 52, 14761 (1995).

${ }^{18}$ K. Flensberg and B. Y.-K. Hu, Phys. Rev. Lett. 73, 3572
(1994)

19 A. Levchenko and A. Kamenev, Phys. Rev. Lett. 100, 026805 (2008).

20 A. F. Andreev, Sov. Phys. JETP Lett. 28, 557 (1978); A. F. Andreev and Yu. A. Kosevich, Sov. Phys. JETP 50, 1218 (1979).

21 B. Spivak, S. V. Kravchenko, S. A. Kivelson, and X. P. A. Gao, Rev. Mod. Phys. 82, 1743 (2010).

22 To be clear, we consider a large $r_{s}$ limit but still assume its value to be smaller than a critical one $r_{s} \lesssim r_{s}^{(c)} \sim 40$ when electron fluid crystallizes into the Wigner state ${ }^{32}$. At a finite temperature the condition is less stringent because the Wigner crystal may be melted by thermal fluctuations.

23 E. H. Hwang, S. Das Sarma, V. Braude, and A. Stern, Phys. Rev. Lett. 90, 086801 (2003).

24 V. M. Apalkov and M. E. Raikh, Phys. Rev. B 71, 245109 (2005).

25 B. Spivak and S. A. Kivelson, Phys. Rev. B 72, 045355 (2005).

26 A. V. Andreev, S. A. Kivelson, and B. Spivak, Phys. Rev. Lett. 106, 256804 (2011).

27 L. D. Landau and E. M. Lifshitz, Sov. Phys. JETP 5, 511 (1958).

28 L. P. Pitaevskii and E. M. Lifshitz, Statistical Physics, Part 2: Volume 9 Course of Theoretical Physics, (Pergamon Press, NY 1980).

29 A. F. Andreev and A. E. Meierovich, JETP Lett. 15, 39 (1972).

30 X. P. A. Gao, G. S. Boebinger, A. P. Mills Jr., A. P. Ramirez, L. N. Pfeiffer, and K. W. West, Phys. Rev. Lett. 94, 086402 (2005).

31 T. J. Gramila, J. P. Eisenstein, A. H. MacDonald, L. N. Pfeiffer and K. W. West, Phys. Rev. B 47, 12957 (1993).

32 B. Tanatar and D. M. Ceperley, Phys. Rev. B 39, 5005 (1989).

33 B. Spivak and S. Kivelson, Ann. Phys. (N.Y.) 321, 2071 (2006).

34 A. A. Abrikosov and I. M. Khalatnikov, Rep. Prog. Phys. 22, 329 (1959). 